\title{
Evidence for a Postsecretory Reabsorptive Site for Uric Acid in Man
}

\author{
Herbert S. Diamond and James S. Paolino \\ From the Department of Medicine, State University of New York Downstate \\ Medical Center, Brooklyn, New York 11203
}

A в S T RACT The effects of administration of drug combinations on uric acid excretion were studied in order to test the hypothesis that a portion of renal tubular reabsorption of uric acid occurs distal to the uric acid secretory site.

Oral administration of pyrazinamide ( $3 \mathrm{~g})$ during probenecid uricosuria (probenecid $500 \mathrm{mg}$ every $6 \mathrm{~h}$ ) decreased urate excretion from $463 \mu \mathrm{g} / \mathrm{min}$ following probenecid medication alone to $135 \mu \mathrm{g} / \mathrm{min}$ following probenecid plus pyrazinamide $(P<0.01)$. When a greater uricosuric effect was induced with a $2 \mathrm{~g}$ oral dose of probenecid, the decrement in urate excretion which followed pyrazinamide administration ( $3 \mathrm{~g}$ ) was more pronounced $(2,528 \mu \mathrm{g} / \mathrm{min}$ following probenecid alone, $574 \mu \mathrm{g} / \mathrm{min}$ following probenecid plus pyrazinamide). Results were similar when an $800 \mathrm{mg}$ oral dose of sulfinpyrazone was given in place of probenecid $(1,885 \mu \mathrm{g} / \mathrm{min}$ following sulfinpyrazone alone, $475 \mu \mathrm{g} / \mathrm{min}$ following sulfinpyrazone plus pyrazinamide). Thus, apparent urate secretion (measured as the decrease in excretion of urinary uric acid resulting from pyrazinamide administration) appeared to vary, depending upon the degree of inhibition of reabsorption produced by probenecid or sulfinpyrazone.

When small doses of aspirin were administered in place of pyrazinamide to produce secretory inhibition, the results were similar.

Neither probenecid nor pyrazinamide significantly altered urate excretion when administered to patients with serum salicylate levels above $14 \mathrm{mg} / 100 \mathrm{ml}$.

These results are interpreted as suggesting that renal tubular reabsorption of uric acid occurs at least in part at a postsecretory site and that a portion of secreted urate is reabsorbed. During maximum probenecid- or sulfinpyrazone-induced uricosuria, inhibition of urate secre-

This work was presented in part before the American Rheumatism Association in Dallas, Texas on 8 June 1972.

Received for publication 18 October 1972 and in revised form 5 February 1973. tion with either pyrazinamide or low doses of aspirin resulted in a decrease in uric acid excretion which exceeded total urinary uric acid during control periods by two- to fourfold. This suggests that renal tubular secretion of urate may greatly exceed uric acid excretion and that a large fraction of secreted urate is reabsorbed.

The pyrazinamide suppression test underestimates urate secretion. Uricosuria induced by some drugs, including probenecid, sulfinpyrazone, and iodinated radioopaque dyes, appears to represent, at least in part, inhibition of postsecretory urate reabsorption.

\section{INTRODUCTION}

Urinary excretion of uric acid is thought to be derived from two sources: uric acid filtered at the glomerulus and incompletely reabsorbed, and uric acid secreted by the renal tubules $(1,2)$. Techniques for determining the relative contribution to total urinary uric acid of each of these mechanisms depend upon the assumption that the site of uric acid reabsorption in the renal tubule is proximal to the site of its secretion $(3,4)$. Recently, however, total urinary uric acid excretion has been reported to increase when urine flow rate increases, due to decreased distal tubular reabsorption of uric acid (5). Recent studies in the rat have provided evidence for distal tubular urate reabsorption in this species (6).

If renal tubular reabsorption of uric acid in man occurs at a site distal to the secretion site, part of what is secreted can be reabsorbed downstream. Under these circumstances, the amount of uric acid in the urine that is attributed to secretion is really that which is secreted and escapes reabsorption. Blocking reabsorption would lead to an apparent increase in the secreted fraction. However, if the reabsorption site is proximal to the secretion site, none of the urate added to the urine by secretion can be reabsorbed and blockade of the reabsorption site would not lead to an increase in the apparently secreted fraction. 
To test the hypothesis that a portion of uric acid reabsorption occurs distal to the secretory site, the effects on uric acid excretion of the administration of drug combinations were studied. Probenecid or sulfinpyrazone was used to block uric acid reabsorption $(7,8)$, pyrazinamide or small-dose aspirin was used to inhibit uric acid secretion $(3,9,10)$, and large-dose aspirin to block both secretion and reabsorption (9). Inhibition of reabsorption of uric acid produced an apparent increase in its secretion. These results are not in accord with the hypothesis that most or all of uric acid reabsorption occurs proximal to the secretory site of uric acid. They are more consistent with the existence of a postsecretory reabsorptive site for uric acid in man.

\section{METHODS}

The subjects selected for investigation were 15 men and one woman ranging in age from $25 \mathrm{yr}$ to $64 \mathrm{yr}$ (mean age, 48). 11 subjects were hyperuricemic and 9 of them had clinical gout. Five subjects were normouricemic controls. All studies were conducted in the morning following an overnight fast. All subjects were at rest and clinically well during the studies. Medication known to effect the level of serum uric acid or urinary uric acid excretion was withdrawn at least 4 days prior to the studies. All subjects were maintained on a purine-free, low-protein, isocaloric diet for 3 days prior to and throughout the studies. Standard renal clearance techniques were employed.

Results in this study were similar for hyperuricemic and normouricemic subjects and the results were therefore all grouped together. This might be questioned since some patients with gout have been reported to have deficient urate secretion (11). However, the differences in urate transport between normal and gouty subjects in these studies were small when compared with the drug-induced changes here reported, and would not be expected to influence the results of the present study.

Glomerular filtration rate was estimated on the basis of endogenous creatinine clearance. Creatinine was determined by AutoAnalyzer method (Technicon Corp., Tarrytown, N. Y.). Uric acid was determined by an automated enzymatic spectrophometric technique (12). Salicylate was determined by the method of Keller (13).

24-h urine collections. $24 \mathrm{~h}$ urinary uric acid excretion, while subjects ate a purine-free diet, was determined by collection of urine for two or three consecutive $24 \mathrm{~h}$ periods. Blood samples were obtained at the beginning and end of each $24-\mathrm{h}$ period. Similar 24 -h urine collections were obtained from subjects receiving probenecid $500 \mathrm{mg}$ every 6 $\mathrm{h}$ alone, or probenecid in combination with salicylate in small dose (300 mg every $6 \mathrm{~h}$ ) or large dose (900-1,200 $\mathrm{mg}$ every $4-6 \mathrm{~h}$ ), or salicylate alone in the small or large dose.

Pyrazinamide studies. The effect of pyrazinamide on urate excretion was determined using a format adapted from that described by Steele and Reiselbach (3). The control phase of each study involved three clearance periods of approximately $20 \mathrm{~min}$ each. Blood for determination of uric acid and creatinine was collected at the start of the initial period and the end of the third period. Total excretion during the three clearance periods was used for calculation of clearances. At the end of the third clearance period, each subject took $3 \mathrm{~g}$ of pyrazinamide orally. $1 \mathrm{~h}$ later, the first of three additional $20 \mathrm{~min}$ clearance periods was begun. Uric acid and creatinine excretion and clearance were calculated as before. Where appropriate, urine collections were continued for $2 \mathrm{~h}$ or longer with blood collections at the beginning and end of each clearance period. Blood for uric acid and creatinine was collected hourly throughout. Maximum decrease in urinary uric acid content usually occurred within $80 \mathrm{~min}$ of pyrazinamide administration, and as previously observed (14), persisted for at least $2 \frac{1}{2} \mathrm{~h}$.

The effect of pyrazinamide on probenecid induced uricosuria. In six subjects, probenecid uricosuria was established by administering probenecid, $500 \mathrm{mg}$ every $6 \mathrm{~h}$ ( 8 a.m., 2 p.m., 8 p.m., and 2 a.m.) for $24-48$ h. On the 2 nd or 3 rd day of probenecid administration, the 8 a.m. dose of probenecid was administered, and then beginning at 9 a.m., the effect of pyrazinamide was determined according to the protocol already described beginning with control clearance periods. Blood samples were obtained hourly during the clearance studies. In three subjects, additional studies were carried out in order to assess the effect of pyrazinamide on maximal probenecid-induced uricosuria. Probenecid, $2 \mathrm{~g}$, was administered as a single oral dose at 9 a.m. on 2 consecutive days, 1 day alone, and the other day in combination with $3 \mathrm{~g}$ of pyrazinamide. Beginning at 9 a.m., four consecutive urine collections, of approximately $30 \mathrm{~min}$ each, were carried out for measurement of uric acid and creatinine clearances. Blood samples were obtained at the beginning and end of each clearance period.

The effect of small doses of aspirin on probenecid-induced uricosuria. In six subjects, $24 \mathrm{~h}$ urinary uric acid excretion was measured both during oral administration of probenecid, $500 \mathrm{mg}$ every $6 \mathrm{~h}$, and during oral administration of probenecid $500 \mathrm{mg}$ every $6 \mathrm{~h}$, together with aspirin $300 \mathrm{mg}$ every 6 h ( 8 a.m., 2 p.m., 8 p.m., and 2 a.m.). In two subjects, the effect of aspirin on maximal probenecid-induced uricosuria was studied by the administration of a single oral $2 \mathrm{~g}$ dose of probenecid at $9 \mathrm{a} . \mathrm{m}$. on 2 consecutive days, 1 day alone, and the other day in combination with $300 \mathrm{mg}$ of aspirin. Blood and urine samples were collected as already described for probenecid and pyrazinamide.

The effect of pyrazinamide on salicylate-induced uricosuria. In order to investigate the interaction of pyrazinamide and aspirin on uric acid excretion, aspirin $20 \mathrm{mg} / \mathrm{kg}$ was administered orally at 10 a.m. on 2 consecutive days, following base-line urine collections. Urine was collected at 30 min intervals for the next $3 \mathrm{~h}$. Blood for determination of serum uric acid, creatinine, and salicylate was collected at the start of the study and at the end of each urine collection. On either the first or the second of the 2 days, pyrazinamide, $3 \mathrm{~g}$, was administered orally $1 \mathrm{~h}$ before administration of aspirin.

Since a single large dose of aspirin often failed to produce uricosuric serum salicylate levels, pyrazinamide was administered to three subjects on the second day of large dose oral salicylate administration (3.6-5.4 g/day in divided doses).

Urine collection. Urinary bladders were not catheterized. In studies involving brief urine collection periods, subjects were hydrated orally with water in amounts sufficient to provide a steady rate of urine flow of at least $5 \mathrm{ml} / \mathrm{min}$. When multiple studies were carried out in a single subject, water intake was identical for all studies so that urine flow rates were comparable. If bladder emptying were incomplete, this could introduce an error in the calculated urate excretion and clearance, particularly in data involving brief urine collection periods. Completeness and accuracy of urine collection were assessed by measurement of creatinine in all 
TABLE I

Effect of Acute Oral Administration of Pyrazinamide $(3 \mathrm{~g})$ on Probenecid Uricosuria (Probenecid $500 \mathrm{mg}$ every $6 \mathrm{~h}$ )

\begin{tabular}{|c|c|c|c|c|c|c|c|c|}
\hline \multirow[b]{2}{*}{ Subject } & \multicolumn{3}{|c|}{ Probenecid alone } & \multirow[b]{2}{*}{ GFR* } & \multicolumn{3}{|c|}{ Probenecid plus pyrazinamide } & \multirow[b]{2}{*}{ GFR } \\
\hline & $\begin{array}{c}\text { Urinary } \\
\text { urate }\end{array}$ & $\begin{array}{l}\text { Serum } \\
\text { urate }\end{array}$ & $\begin{array}{c}\text { Urate } \\
\text { clearance }\end{array}$ & & $\begin{array}{l}\text { Urinary } \\
\text { urate }\end{array}$ & $\begin{array}{l}\text { Serum } \\
\text { urate }\end{array}$ & $\begin{array}{c}\text { Urate } \\
\text { clearance }\end{array}$ & \\
\hline & $\mu g / \min$ & $m g / 100 m l$ & $m l / m i n$ & $m l / m i n$ & $\mu g / \min$ & $m g / 100 m l$ & $m l / \min$ & $m l / m i n$ \\
\hline H. S. & 212 & 7.4 & 2.9 & 123 & 49 & 7.6 & 0.6 & 130 \\
\hline H. D. & 638 & 5.9 & 10.8 & 129 & 137 & 5.8 & 2.3 & 132 \\
\hline L. K. & 587 & 5.5 & 10.7 & 126 & 163 & 5.6 & 2.4 & 132 \\
\hline A. M. & 622 & 3.0 & 20.5 & 121 & 200 & 3.3 & 6.0 & 118 \\
\hline $\mathrm{O} . \mathrm{P}$. & 375 & 5.6 & 6.7 & 75 & 162 & 6.0 & 2.7 & 105 \\
\hline J.S. & 345 & 5.5 & 6.3 & 103 & 98 & 6.7 & 1.5 & 86 \\
\hline Mean & 463 & 5.5 & 9.7 & 113 & $135 \ddagger$ & 5.8 & $2.6 \S$ & 117 \\
\hline
\end{tabular}

* Glomerular filtration rate.

† Significantly different from urinary urate following probenecid alone $(P<0.01)$.

$\S$ Significantly different from urate clearance following probenecid alone $(P<0.05)$.

urine collections. Data from collection periods in which urinary creatinine determinations indicated significant error in urine collections are not included in this report. Urinary excretion of urate was calculated both as total excretion and as urate excretion per creatinine clearance and urate clearance per creatinine clearance. Since these latter calculations are based only on urine and plasma concentrations,

TABLE IIA

Effect of Acute Administration of Probenecid $(2 \mathrm{~g})$ or Sulfinpyrazone $(800 \mathrm{mg})$ on Uric Acid Excretion

\begin{tabular}{|c|c|c|c|c|}
\hline Subject & Time & $\begin{array}{l}\text { Urine } \\
\text { urate }\end{array}$ & $\begin{array}{l}\text { Serum } \\
\text { urate }\end{array}$ & $\begin{array}{c}\text { Urate } \\
\text { clearance }\end{array}$ \\
\hline & $\min$ & $\mu g / \min$ & $\mathrm{mg} / 100 \mathrm{ml}$ & $\mathrm{ml} / \mathrm{min}$ \\
\hline L. S. & $0-30$ & 350 & 11.0 & 3.2 \\
\hline \multicolumn{5}{|c|}{ Probenecid orally } \\
\hline & $30-60$ & 527 & 11.0 & 4.8 \\
\hline & 60-90 & 1680 & 10.6 & 15.8 \\
\hline & $90-150$ & 2983 & 10.3 & 29.0 \\
\hline \multirow[t]{2}{*}{ H. D. } & $0-30$ & 554 & 5.8 & 9.6 \\
\hline & $30-60$ & 528 & 5.7 & 9.3 \\
\hline \multicolumn{5}{|c|}{ Probenecid orally } \\
\hline & $60-90$ & 648 & 5.8 & 11.2 \\
\hline & $90-120$ & 1526 & 5.7 & 26.8 \\
\hline & $120-150$ & 2872 & 5.4 & 53.1 \\
\hline & $150-180$ & 3340 & 5.1 & 65.5 \\
\hline \multirow[t]{2}{*}{ A. P. } & $0-30$ & 313 & 4.7 & 6.7 \\
\hline & $30-60$ & 346 & 4.8 & 7.2 \\
\hline \multicolumn{5}{|c|}{ Probenecid orally } \\
\hline & 60-90 & 412 & 4.6 & 9.0 \\
\hline & $90-120$ & 729 & 4.5 & 16.2 \\
\hline & $120-150$ & 1261 & 4.3 & 29.3 \\
\hline & $150-180$ & 1129 & 3.8 & 30.0 \\
\hline
\end{tabular}

they would not be affected by small errors in urine collection. Factoring of urate excretion or clearance by glomerular filtration rate did not alter the results.

TABLE IIB

Effect of Acute Administration of Probenecid (2 g) or Sulfinpyrazone in Combination with Pyrazinamide $(3 \mathrm{~g})$ on Uric Acid Excretion

\begin{tabular}{|c|c|c|c|c|c|}
\hline Subject & Time & $\begin{array}{l}\text { Urine } \\
\text { urate }\end{array}$ & $\begin{array}{c}\text { Serum } \\
\text { urate }\end{array}$ & $\begin{array}{c}\text { Urate } \\
\text { clearance }\end{array}$ & $\begin{array}{c}\text { Decrease } \\
\text { in urine } \\
\text { urate after } \\
\text { pyrazinamide }\end{array}$ \\
\hline \multirow{6}{*}{ L. S. } & $\min$ & $\mu g / \min$ & $\begin{array}{c}\mathrm{mg} / \\
100 \mathrm{ml}\end{array}$ & $m l / m i n$ & $\mu g / \min$ \\
\hline & $0-30$ & 411 & 6.6 & 6.2 & +61 \\
\hline & \multicolumn{5}{|c|}{ Probenecid and pyrazinamide orally } \\
\hline & $30-60$ & 462 & 6.5 & 7.1 & -65 \\
\hline & $60-90$ & 691 & 6.4 & 10.8 & -989 \\
\hline & $90-165$ & 480 & 6.4 & 7.5 & $-2,503$ \\
\hline \multirow[t]{2}{*}{ H. D. } & $0-30$ & 522 & 6.1 & 8.6 & -32 \\
\hline & $30-60$ & 538 & 6.1 & 8.8 & +10 \\
\hline
\end{tabular}

Probenecid and pyrazinamide orally

$\begin{array}{cccrr}60-90 & 542 & 6.0 & 9.0 & -106 \\ 90-120 & 618 & 6.1 & 10.1 & -908 \\ 120-150 & 603 & 5.9 & 10.2 & -2,269 \\ 150-180 & 656 & 5.8 & 11.3 & -2,684 \\ 0-30 & 329 & 5.0 & 6.6 & +16 \\ 30-60 & 303 & 4.9 & 6.2 & -43\end{array}$

Probenecid and pyrazinamide orally

$\begin{array}{ccccr}60-90 & 365 & 5.0 & 7.3 & -47 \\ 90-120 & 374 & 5.0 & 7.5 & -355 \\ 120-150 & 286 & 4.9 & 5.8 & -975 \\ 150-180 & 315 & 4.8 & 6.6 & -814\end{array}$


TABLE IIIA

Effect of Acute Administration of Sulfinpyrazone (800 mg) Alone on Uric Acid Excretion

\begin{tabular}{|c|c|c|c|c|}
\hline Subject & Time & $\begin{array}{l}\text { Urine } \\
\text { urate }\end{array}$ & $\begin{array}{l}\text { Serum } \\
\text { urate }\end{array}$ & $\begin{array}{l}\text { Urate } \\
\text { clearance }\end{array}$ \\
\hline & $\min$ & $\mu g m / \min$ & $m g / 100 m l$ & $m l / \min$ \\
\hline \multirow[t]{6}{*}{ H. M. } & $0-60$ & 124 & 9.3 & 1.3 \\
\hline & \multicolumn{3}{|c|}{ Sulfinpyrazone orally } & \\
\hline & $60-90$ & 215 & 9.5 & 2.3 \\
\hline & $90-120$ & 285 & 9.4 & 3.0 \\
\hline & $120-150$ & 550 & 8.9 & 6.2 \\
\hline & $150-180$ & 867 & 8.3 & 10.4 \\
\hline \multirow[t]{6}{*}{ H. D. } & $0-60$ & 544 & 5.6 & 9.7 \\
\hline & \multicolumn{3}{|c|}{ Sulfinpyrazone orally } & \\
\hline & $60-90$ & 734 & 5.7 & 12.9 \\
\hline & $90-120$ & 1,811 & 5.7 & 31.7 \\
\hline & $120-150$ & 2,836 & 5.5 & 51.6 \\
\hline & $150-180$ & 2,902 & 5.1 & 56.9 \\
\hline
\end{tabular}

Statistical analysis. Results are expressed as mean and standard error of the mean. Statistical significance was determined by a $t$ test of paired variables. Differences were considered significant where $P<0.05$.

\section{RESULTS}

Effect of oral administration of pyrazinamide on probenecid- or sulfinpyrazone-induced uricosuria. Uricosuria was established in six subjects by the oral administration of probenecid $2 \mathrm{~g}$ daily. The acute oral administration of pyrazinamide $3 \mathrm{~g}$ during probenecid uricosuria decreased urate excretion from a mean of $463 \pm$

TABLE IIIB

Effect of Acute Administration of Sulfinpyrazone $(800 \mathrm{mg}$ ) with Pyrazinamide $(3 \mathrm{~g})$ on Uric Acid Excretion

\begin{tabular}{|c|c|c|c|c|c|}
\hline Subject & Time & $\begin{array}{l}\text { Urine } \\
\text { urate }\end{array}$ & $\begin{array}{l}\text { Serum } \\
\text { urate }\end{array}$ & $\begin{array}{c}\text { Urate } \\
\text { clearance }\end{array}$ & $\begin{array}{c}\text { Decrease } \\
\text { in urine } \\
\text { urate after } \\
\text { pyrazinamide }\end{array}$ \\
\hline & $\min$ & $\mu g / \min$ & $\begin{array}{c}m g / \\
100 m l\end{array}$ & $\mathrm{ml} / \mathrm{min}$ & $\mu g m / \min$ \\
\hline \multirow[t]{6}{*}{ H. M. } & $0-60$ & 47 & 8.0 & 0.6 & -77 \\
\hline & Sulfinpyra & one $+p$ & yrazina & mide oral & \\
\hline & 60-90 & 18 & 8.0 & 0.2 & -197 \\
\hline & $90-120$ & 14 & 8.0 & 0.2 & -271 \\
\hline & $120-150$ & 22 & 8.2 & 0.3 & -528 \\
\hline & $150-180$ & 79 & 8.1 & 1.0 & -788 \\
\hline \multirow[t]{5}{*}{ H. P. } & $0-60$ & 603 & 5.8 & 10.4 & +59 \\
\hline & 60-90 & 497 & 5.6 & 8.9 & -237 \\
\hline & $90-120$ & 430 & 5.6 & 7.7 & $-1,381$ \\
\hline & $120-150$ & 569 & 5.6 & 10.2 & $-2,267$ \\
\hline & $150-180$ & 871 & 5.7 & 15.3 & $-2,031$ \\
\hline
\end{tabular}

$72 \mu \mathrm{g} / \mathrm{min}$ when probenecid alone was given to $135 \pm 21$ $\mu \mathrm{g} / \mathrm{min}$ when probenecid was given with pyrazinamide $(P<0.01)$ (Table I). In all six subjects, there was little change in serum urate level. Urate clearance decreased from $9.7 \pm 2.5 \mathrm{ml} / \mathrm{min}$ when probenecid was given alone to $2.6 \pm 0.8 \mathrm{ml} / \mathrm{min}$ following administration of probenecid plus pyrazinamide $(P<0.05)$. Pyrazinamide administration reversed probenecid uricosuria, resulting in urate excretion which was less than control urate excretion when no drug was administered.

The decrement in urate excretion produced by pyrazinamide was more pronounced when profound uricosuria was induced by oral administration of either a single $2 \mathrm{~g}$ dose of probenecid (Table II), or an $800 \mathrm{mg}$ dose of sulfinpyrazone (Table III). Urate excretion increased from a mean of $407 \mu \mathrm{g} / \mathrm{min}$ when no drug was given to peak of $2,528 \mu \mathrm{g} / \mathrm{min}$ following administration of probenecid alone. Maximum urate excretion was only 574 $\mu \mathrm{g} / \mathrm{min}$ when pyrazinamide was administered together with probenecid. Similarly, urate excretion increased from a mean of $334 \mu \mathrm{g} / \mathrm{min}$ when no drug was given to a peak of $1,885 \mu \mathrm{g} / \mathrm{min}$ following administration of sulfinpyrazone alone. Maximum urate excretion was only $475 \mu \mathrm{g} / \mathrm{min}$ when pyrazinamide was administered together with sulfinpyrazone. The decrease in urate excretion attributed to pyrazinamide at the time of maximal probenecid or sulfinpyrazone effect, was four times as large as urate excretion when no drug was taken. If pyrazinamide effect is attributed to inhibition of renal tubular urate secretion, then urate secretion must have been larger than total excretion of uric acid in the urine of subjects who were taking no medication. Thus, apparent renal tubular urate secretion (measured as the decrease in urinary uric acid resulting from pyrazinamide administration) appeared to vary, dependent upon

TABLE IV

Effect of Salicylate on 24 h Urinary Uric Acid Excretion during Probenecid Administration

\begin{tabular}{lcccc}
\hline & & \multicolumn{3}{c}{$24 \mathrm{~h}$ Urinary uric acid } \\
\cline { 3 - 5 } Subject & No drug & $\begin{array}{c}\text { Probenecid } \\
(500 \mathrm{mg} \\
\text { every } 6 \mathrm{~h})\end{array}$ & $\begin{array}{c}\text { Salicylate } \\
(300 \mathrm{mg} \\
\text { every } 6 \mathrm{~h})\end{array}$ & $\begin{array}{c}\text { Probenecid } \\
\text { plus } \\
\text { salicylate }\end{array}$ \\
\hline & $m g / 24 h$ & $m g / 24 h$ & $m g / 24 h$ & $m g / 24 h$ \\
H. S. & 614 & 875 & 326 & 528 \\
J. S. & 524 & 837 & 428 & 796 \\
L. S. & 507 & 705 & 278 & 435 \\
H. D. & 573 & 848 & 465 & 562 \\
O. P. & 454 & 637 & 387 & 392 \\
R. M. & 724 & 770 & 355 & 464 \\
Mean & 566 & 779 & 373 & 530 \\
SE & \pm 39 & \pm 38 & \pm 28 & \pm 59 \\
\hline
\end{tabular}


TABLE V A

Effect of Acute Oral Administration of Salicylate $(300 \mathrm{mg})$ on Urate Excretion Induced by Probenecid $(2 \mathrm{~g})$

\begin{tabular}{|c|c|c|c|c|}
\hline \multirow[b]{2}{*}{ Subject } & \multirow[b]{2}{*}{ Time } & \multicolumn{3}{|c|}{ Probenecid alone } \\
\hline & & $\begin{array}{l}\text { Urine } \\
\text { urate }\end{array}$ & $\begin{array}{l}\text { Serum } \\
\text { urate }\end{array}$ & $\begin{array}{c}\text { Urate } \\
\text { clearance }\end{array}$ \\
\hline \multirow[b]{2}{*}{ H. D. } & $\min$ & $\mu g m / \min$ & $m g / 100 m l$ & $m l / m i n$ \\
\hline & $\begin{array}{r}0-30 \\
30-60\end{array}$ & $\begin{array}{l}554 \\
528\end{array}$ & $\begin{array}{l}5.8 \\
5.7\end{array}$ & $\begin{array}{l}9.6 \\
9.3\end{array}$ \\
\hline \multicolumn{5}{|c|}{ Probenecid orally } \\
\hline & $\begin{array}{c}60-90 \\
90-120 \\
120-150 \\
150-180\end{array}$ & $\begin{array}{r}648 \\
1,526 \\
2,872 \\
3,340\end{array}$ & $\begin{array}{l}5.8 \\
5.7 \\
5.4 \\
5.1\end{array}$ & $\begin{array}{l}11.2 \\
26.8 \\
53.1 \\
65.5\end{array}$ \\
\hline L.S. & $0-30$ & 350 & 11.0 & 3.2 \\
\hline \multicolumn{5}{|c|}{ Probenecid orally } \\
\hline & $\begin{array}{l}30-60 \\
60-90 \\
90-150\end{array}$ & $\begin{array}{r}527 \\
1,680 \\
2,983\end{array}$ & $\begin{array}{l}11.0 \\
10.6 \\
10.3\end{array}$ & $\begin{array}{r}4.8 \\
15.8 \\
29.0\end{array}$ \\
\hline
\end{tabular}

the degree of inhibition of uric acid reabsorption produced by probenecid.

Effect of small doses of aspirin on probenecid-induced uricosuria. When small doses of aspirin were administered to produce inhibition of urate secretion, the results were similar to those when urate secretion was inhibited with pyrazinamide (Table IV). Mean $24 \mathrm{~h}$ urate excretion decreased from $779 \pm 38 \mathrm{mg}$ while probenecid $2 \mathrm{~g} /$ day was given alone to $530 \pm 59 \mathrm{mg}$ when probenecid was administered along with aspirin $300 \mathrm{mg}$ every $6 \mathrm{~h}$ $(P<0.01)$. The mean decrease in urate excretion following small-dose aspirin administration was greater when aspirin was administered during partial inhibition of reabsorption by probenecid $(249 \pm 43 \mu \mathrm{g} / \mathrm{min})$ than during aspirin administration alone $(193 \pm 49 \mu \mathrm{g} / \mathrm{min})$.

When a greater degree on inhibition of urate reabsorption was produced with a single large dose of probenecid, the effect of secretory inhibition by aspirin was more pronounced (Table V). Administration of $300 \mathrm{mg}$ aspirin with $2 \mathrm{~g}$ probenecid resulted in a decrease in maximal urate excretion from $3,162 \mu \mathrm{g} / \mathrm{min}$ when receiving probenecid alone to $1,152 \mu \mathrm{g} / \mathrm{min}$ when probenecid plus aspirin was given. Again the decrement in urate excretion $(2,010 \mu \mathrm{g} / \mathrm{min})$ following inhibition of urate secretion by aspirin exceeded control urate excretion by almost fourfold ( $562 \mu \mathrm{g} / \mathrm{min})$.

Effect of oral administration of pyrazinamide or probenecid on salicylate-induced uricosuria. When a single large dose of aspirin $(20 \mathrm{mg} / \mathrm{kg})$ was administered orally to eight subjects, serum salicylate levels were generally less than $5 \mathrm{mg} / 100 \mathrm{ml}$ at the end of the first 30
TABLE V B

Effect of Acute Oral Administration of Salicylate (300 mg) on Urate Excretion Induced by Probenecid (2 g)

\begin{tabular}{|c|c|c|c|c|c|}
\hline \multirow[b]{2}{*}{ Subject } & \multirow[b]{2}{*}{ Time } & \multicolumn{4}{|c|}{ Probenecid with salicylate } \\
\hline & & $\begin{array}{l}\text { Urine } \\
\text { urate }\end{array}$ & $\begin{array}{c}\text { Serum } \\
\text { urate }\end{array}$ & $\begin{array}{c}\text { Urate } \\
\text { clearance }\end{array}$ & $\begin{array}{l}\text { Decrease } \\
\text { in urine } \\
\text { urate after } \\
\text { salicylate }\end{array}$ \\
\hline \multirow{3}{*}{ H. D. } & $\min$ & $\mu g m / \min$ & $\begin{array}{c}m g / \\
100 m l\end{array}$ & $m l / m i n$ & $\mu g m / \min$ \\
\hline & $0-30$ & 592 & 6.2 & 9.5 & +38 \\
\hline & $30-60$ & 553 & 6.3 & 8.8 & +25 \\
\hline \multicolumn{6}{|c|}{ Probenecid and salicylate orally } \\
\hline \multirow{9}{*}{ L. S. } & $60-90$ & 589 & 6.4 & 9.2 & -59 \\
\hline & $90-120$ & 885 & 6.3 & 14.0 & -641 \\
\hline & $120-150$ & 1,354 & 6.1 & 22.2 & $-1,518$ \\
\hline & $150-180$ & 1,431 & 5.8 & 24.7 & $-1,909$ \\
\hline & $0-30$ & 416 & 9.2 & 4.5 & +66 \\
\hline & \multicolumn{4}{|c|}{ Probenecid and salicylate orally } & \\
\hline & $30-60$ & 525 & 9.2 & 5.7 & -2 \\
\hline & $60-90$ & 794 & 9.0 & 8.8 & -886 \\
\hline & $90-150$ & 873 & 8.8 & 9.9 & $-2,110$ \\
\hline
\end{tabular}

min clearance period and increased to peak values of $8-22 \mathrm{mg} / 100 \mathrm{ml}$ at $90-120 \mathrm{~min}$ after drug administration. The low serum salicylate levels in the first clearance period after aspirin ingestion were associated with a decrease in urate excretion from $320 \pm 76 \mu \mathrm{g} / \mathrm{min}$ when no drug was given, to $217 \pm 56 \mu \mathrm{g} / \mathrm{min}$ after aspirin. Although the response in uric acid excretion following salicylate administration varied considerably from subject to subject, uricosuria usually occurred when peak serum salicylate levels exceeded $14 \mathrm{mg} / 100 \mathrm{ml}$.

The biphasic response to aspirin administration of urate retention, at low serum salicylate level and uricosuria at high serum salicylate level, is attributed to secretory inhibition by lesser concentrations of aspirin and inhibition of both secretion and reabsorption by greater concenrations of aspirin (9). Large doses of aspirin presumably result in almost total inhibition of urate secretion and nearly maximal inhibition of urate reabsorption. If this analysis be correct, then, in the presence of large doses of salicylate, administration of either pyrazinamide (to block secretion) or probenecid (to block reabsorption) should have little effect on urate excretion, since both secretion and reabsorption are already blocked.

The effect of pyrazinamide on urate excretion in subjects with serum salicylate levels greater than $14 \mathrm{mg} / 100$ $\mathrm{ml}$ is summarized in Table VI. High serum salicylate levels were achieved by administration of a single dose salicylate $20 \mathrm{mg} / \mathrm{kg}$ orally (subjects $1-4$ ), or by adminis- 
TABLE VI

Effect of Acute Oral Administration of Pyrazinamide $(3 \mathrm{~g})$ on Salicylate-Induced Uricosuria

\begin{tabular}{|c|c|c|c|c|c|c|}
\hline \multirow[b]{2}{*}{ Subject } & \multicolumn{3}{|c|}{ Salicylate alone } & \multicolumn{3}{|c|}{ Salicylate plus pyrazinamide } \\
\hline & $\begin{array}{l}\text { Urinary } \\
\text { urate }\end{array}$ & $\begin{array}{l}\text { Serum } \\
\text { urate }\end{array}$ & $\begin{array}{c}\text { Serum } \\
\text { salicylate }\end{array}$ & $\begin{array}{l}\text { Urinary } \\
\text { urate }\end{array}$ & $\begin{array}{c}\text { Serum } \\
\text { urate }\end{array}$ & $\begin{array}{c}\text { Serum } \\
\text { salicylate }\end{array}$ \\
\hline & $\mu g / \min$ & $\mathrm{mg} / 100 \mathrm{ml}$ & $\mathrm{mg} / 100 \mathrm{ml}$ & $\mu g / \min$ & $\mathrm{mg} / 100 \mathrm{ml}$ & $\mathrm{mg} / 100 \mathrm{ml}$ \\
\hline S. H. & 683 & 5.4 & 14 & 480 & 5.4 & 14 \\
\hline R. L. & 220 & 6.2 & 14 & 500 & 7.4 & 17 \\
\hline E. W. & 55 & 8.3 & 16 & 140 & 8.0 & 18 \\
\hline H. D. & 517 & 6.7 & 17 & 654 & 6.4 & 22 \\
\hline A. C. & 356 & 9.9 & 18 & 263 & 9.2 & 16 \\
\hline O. P. & 854 & 5.2 & 21 & 700 & 5.1 & 19 \\
\hline K. M. & 565 & 6.8 & 24 & 425 & 7.1 & 21 \\
\hline Mean & 464 & 6.9 & 17.7 & 451 & 6.9 & 18.1 \\
\hline SE & \pm 104 & \pm 0.6 & \pm 1.4 & \pm 75 & \pm 0.6 & \pm 1.1 \\
\hline
\end{tabular}

tration of aspirin 3.6-5.4 g/day for 1-2 days (subjects $5-7$ ). In seven subjects, mean urate excretion was 464 $\pm 104 \mu \mathrm{g} / \mathrm{min}$ when the large doses of aspirin alone were given and $452 \pm 75 \mu \mathrm{g} / \mathrm{min}$ when pyrazinamide was added. Similarly, when probenecid $500 \mathrm{mg}$ every $6 \mathrm{~h}$ was added to aspirin at a dosage of $3.6-6.4 \mathrm{~g} / \mathrm{day}$, urate excretion did not change (Table VII).

\section{DISCUSSION}

A study employing combinations of drugs with known individual effects on uric acid excretion has been used as a means of quantitatively estimating the effect inhibition of urate reabsorption has on the component of urinary uric acid apparently secreted by the renal tubule. The interpretation of the results obtained is based in part on assumptions regarding the most probable mechanisms of action of the drugs employed.

The decrease in total urate excretion which follows pyrazinamide administration is assumed to represent

TABLE VII

Effect of Probenecid (500 mg every 6 h) on Salicylate (3.6-5.4 g/day) Uricosuria $24 \mathrm{~h}$ Urinary Uric Acid

\begin{tabular}{lccc}
\hline & & & $\begin{array}{c}\text { Salicylate } \\
\text { plus } \\
\text { probenecid }\end{array}$ \\
\hline H. S. & 614 & 914 & 930 \\
J. S. & 524 & 714 & 796 \\
H. D. & 573 & 695 & 722 \\
O. P. & 454 & 492 & 620 \\
R. M. & 724 & 737 & 748 \\
Mean & 578 & 710 & 763 \\
SE & \pm 45 & \pm 67 & \pm 51 \\
\hline
\end{tabular}

essentially complete suppression of renal tubular secretion of urate with little or no effect on urate filtration or reabsorption. Uric acid appearing in the urine following administration of pyrazinamide is assumed to represent uric acid filtered at the glomerulus and not reabsorbed. The data supporting these assumptions have previously been summarized $(3,4)$. In the present study, the decrease in urate excretion produced by pyrazinamide alone was similar to that previously described $(3,4)$.

Several recent animal studies have suggested additional effects of pyrazinamide on tubular transport of urate. Experiments in the cebus monkey and chimpanzee have been interpreted to suggest that in these species, pyrazinamide may increase urate reabsorption by the renal tubule, as well as inhibiting secretion $(15,16)$. More recently, a dual action consisting of inhibition of urate secretion by small doses of pyrazinamide and inhibition of urate reabsorption by large doses of pyrazinamide has been described (17). There was no evidence of inhibition of urate reabsorption by the $3 \mathrm{~g}$ doses of pyrazinamide used in the present study. Partial inhibition of urate reabsorption by pyrazinamide superimposed upon inhibition of urate secretion would not explain the results of the present drug interaction studies. Evidence for a primary mechanism of action of pyrazinamide in man other than inhibition of urate secretion might result in a different interpretation of the results of the present study.

The uricosuric effects of probenecid and sulfinpyrazone are generally accepted as representing inhibition of renal tubular reabsorption of urate $(7,18)$. The uricosuric effect of large doses of salicylate in all probability results from a similar mechanism. The urate-retaining effect of small doses of salicylate might be attributed to stimulation of urate reabsorption by these small doses. However, as pointed out by $\mathrm{Yü}$ and Gutman (9), this 
effect requires the assumption that different dose levels of the same drug can result in either stimulation or inhibition of the same transport mechanism. Thus, the alternative concept of inhibition of urate secretion by small doses of salicylate and inhibition of both urate secretion and reabsorption by large doses of salicylate has generally been accepted. The uricosuric effects of probenecid, sulfinpyrazone, and salicylate and the urate-retaining effects of small doses of salicylate observed in the present study were similar to the results previously reported by others $(7,9)$.

In the present study, during probenecid- or sulfinpyrazone-induced uricosuria, inhibtion of urate secretion by the administration of either pyrazinamide or small doses of salicylate resulted in a marked decrease in amount of uric acid in the urine. The results of these drug interaction studies are consistent with results previously reported by others. Y $\ddot{u}$ and Gutman (9), studying the effect of small doses of salicylate on probenecid-induced uricosuria, noted that the decrease in urate excretion produced by salicylate during probenecid uricosuria could exceed total urinary uric acid and could far exceed the postulated proximal secretion or uric acid. The interaction of sulfinpyrazone with aspirin is similar to the interaction of probenecid with pyrazinamide and aspirin (18).

Fanneli, Bohn, and Riley (16) reported that in the chimpanzee, pyrazinamide produced a decrement in urate excretion during probenecid uricosuria which greatly exceeded total urinary uric acid when no drug was given. Although there is no evidence which would exclude the possibility that pyrazinamide acts in man by stimulating uric acid reabsorption rather than inhibiting its secretion, the similarity of the effects of small doses of aspirin and $3 \mathrm{~g}$ pyrazinamide in the present study and the paradoxical effects of aspirin on urate excretion make this explanation less likely.

A similar biphasic response of urate excretion has also been observed following administration of low and high doses of either probenecid (19) or sulfinpyrazone (8). Thus, high doses of these drugs might be potent inhibitors of urate secretion in man. If probenecid or sulfinpyrazone are potent inhibitors of urate secretion in man, then the profound decrement in urate excretion which follows the administration of pyrazinamide together with one of these drugs could not logically be attributed to inhibition of urate secretion by pyrazinamide. However, at least for sulfinpyrazone, this does not appear to be the case, since tubular secretion of urate has been demonstrated in normal man following the administration of large doses of sulfinpyrazone (20).

Thus, the most direct interpretation of the results of the present study is that urate reabsorption occurs, at least in part, distal to the urate secretory site. Under these circumstances, the decrease in urate excretion which follows inhibition of urate secretion by pyrazinamide is a measure of urate secretion less the portion of this secretion that is reabsorbed. Unless reabsorption of secreted urate is inhibited, pyrazinamide administration does not provide a measure of total urate secretion.

It is recognized that the interpretation of the mechanism producing the effects of drug combinations on uric acid excretion is complex. In addition to effects on urate transport, possible forms of drug interaction include drug-induced alteration of uric acid binding to serum proteins and alteration of serum protein binding, metabolism, or renal transport of one drug by another.

Probenecid and salicylate decrease urate binding to serum proteins (21). However, there is little evidence for significant urate binding to serum proteins under physiologic conditions $(22,23)$. There is no correlation between effects of a drug on urate excretion in a given species and the effects of the same drug on urate binding by serum protein (24). Also, since reabsorption of uric acid is thought to be almost complete at physiologic filtered uric acid loads $(3,4)$, small changes in filtered urate load should have little or no effect on urate excretion.

Competition between drugs for binding sites on serum proteins, as has been demonstrated to occur between salicylate and sulfinpyrazone (18), is possible, but is not likely to be of sufficient magnitude to account for the results here reported.

Pyrazinamide, or its active metabolite, pyrazinoic acid, might competitively inhibit the effect of probenecid and sulfinpyrazone on urate reabsorption. A similar explanation has been offered for the inhibition of sulfinpyrazone uricosuria by low doses of aspirin (18). Since the ability to suppress probenecid- or sulfinpyrazone-induced uricosuria is shown by several drugs which inhibit urate secretion, including aspirin, pyrazinamide, and lactate $(9,25)$, the proposal of an additional effect for these drugs is uneconomical. Moreover, this competitive inhibition of drug effects does not explain the apparent excesive urate secretion (as measured by pyrazinamide suppression) observed when uricosuria resulted from tubular disease (17), sickle cell anemia (26), or water diuresis (5).

Alteration of renal transport of one drug by another or interference with effects on urate transport of one drug by another is an alternative explanation for the drug interactions observed in this study. Probenecid is actively secreted by the proximal tubule (27). If pyrazinamide and small doses of aspirin inhibit probenecid secretion, they might decrease the concentration of probenecid distal to the site where probenecid is secreted. This effect would result in a decreased concentration of probenecid at a urate reabsorptive site distal to the pro- 
benecid secretory site, but would not alter probenecid concentration at a proximal, presecretory, reabsorptive site. Thus, this form of drug interaction would also be consistent with a reabsorptive site for urate distal to its secretory site in the renal tubule. Probenecid is known to be reabsorbed in the distal tubule (27), a finding which is consistent with the hypothesis that at least part of probenecid uricosuria is due to inhibition of urate reabsorption in the distal tubule. Sulfinpyrazone and other phenylbutazone analogues are strongly protein bound and enter the urine almost entirely by secretion, probably at the same site as probenecid (28). These drugs are also reabsorbed in the distal tubule and are markedly uricosuric, probably through inhibition of urate reabsorption. Again the site of inhibition of urate reabsorption in the renal tubule is more likely to be distal to or coextensive with the secretory site for both the drugs and uric acid. Although this interpretation of the present results is plausible, it should be noted that this form of drug interaction would not explain pyrazinamide inhibition of uricosuria, interpreted as apparent increased urate secretion in renal tubular disease (17), sickle cell anemia (26), or following water diuresis (5) when uricosuria was not drug induced.

Thus, either of the two most probable interpretations of the results reported here indicate that urate reabsorption occurs at a site distal to or coextensive with the urate secretory site, and that a portion of secreted urate is reabsorbed. Postsecretory urate reabsorption may be localized to either the proximal or distal tubule or may occur at both sites.

The present study should not be interpreted as suggesting that all reabsorption of urate in the renal tubule occurs distal to the secretory site. Steele (14) has reported an increase in the fraction of urate excretion not inhibited by pyrazinamide under conditions of extracellular fluid volume expansion. This finding suggests that under conditions in which proximal tubular reabsorption of sodium and water is altered, urate reabsorption changes in the same direction as sodium and water reabsorption. Since these alterations in urate excretion do not affect the secretory component, they probably represent proximal presecretory urate reabsorption. If the decrease in urate secretion which follows administration of either pyrazinamide or small doses of salicylate, is attributed to inhibition of urate secretion, then the magnitude of the decrease provides a minimum estimate of renal tubular secretion of uric acid under the conditions of the study. As estimated by pyrazinamide inhibition of secretion, the apparent contribution of urate secretion to uric acid excretion varied dependent upon the degree of inhibition of urate reabsorption produced by probenecid. During maximal drug-induced uricosuria, inhibition of urate secretion with either small doses of aspirin or pyrazin- amide decreased urate excretion by an amount which exceeded by two- to fourfold total urinary uric acid during control periods. This finding is in accord with predictions based on the hypothesis that part or all of urate reabsorption occurs at a site in the renal tubule coextensive with or distal to the urate secretory site and that a portion of secreted urate is reabsorbed. Thus, using this model, the secretory component of uric acid excretion would appear to exceed total urinary uric acid by several fold.

In conclusion, evidence is presented suggesting that renal tubular reabsorption of uric acid occurs at least in part at a site in the tubule distal to coextensive with the site for uric acid secertion, and that a portion of secreted urate is reabsorbed. Tubular secretion of urate appears to be greater than has generally been estimated and may exceed total urinary uric acid by several fold. The pyrazinamide suppression test underestimates urate secretion. The uricosuric effects of some drugs such as probenecid, sulfinpyrazone, and iodinated radioopaque dyes $(29,30)$, are reversed by pyrazinamide. Uricosuria induced by these drugs appears to represent at least in part, inhibition of postsecretory urate reabsorption.

\section{ACKNOWLEDGMENTS}

We thank the nurses and staff of the Clinical Research Center for their efforts in caring for our patients.

This work was supported by grant RR-318 from the General Clinical Research Centers Program of the Division of Research Resources, National Institutes of Health, and a grant from the Arthritis Foundation.

\section{REFERENCES}

1. Gutman, A. B., and T. F. Yu. 1961. Three-component system for regulation of renal excretion of uric acid in man. Trans. Assoc. Am. Physicians (Phila.). 74: 353.

2. Gutman, A. B., T. F. Yü, and L. Berger. 1959. Tubular secretion of urate in man. J. Clin. Invest. 38: 1778.

3. Steele, T. H., and R. E. Rieselbach. 1967. The renal mechanism for urate homeostasis in normal man. Am. J. Med. $43: 868$.

4. Gutman, A. B., T. F. Yu, and L. Berger. 1969. Renal function in gout. III. Estimation of tubular secretion and reabsorption of uric acid by use of pyrazinoic acid (pyrazinamide). Am. J. Med. 47: 575.

5. Diamond, H. S., R. Lazarus, D. Kaplan, and D. Halberstam. 1972. Effect of urine flow rate on uric acid excretion in man. Arthritis Rheum. 15: 338.

6. Gregor, R., F. Lang, and P. Deetjen. 1971. Handling of uric acid by the rat kidney. I. Microanalysis of uric acid in proximal tubular fluid. Pfuegers Arch. 324: 279.

7. Sirota, J. H., T. F. Yu, and A. B. Gutman. 1952. Effect of benemid ( $p$-[di- $n$-propylsulfamyl]-benzoic acid) on urate clearance and other discrete renal functions in gouty subjects. J. Clin. Invest. 31: 692 .

8. Burns, J. J., T. F. Yü, A. Ritterband, J. M. Perel, A. B. Gutman, and B. B. Brodie. 1957 A potent new uricosuric agent, the sulfoxide metabolite of the phenylbutazone analogue, G-25671 J. Pharmacol. Exp. Ther. 119: 418 . 
9. Yü, T. F., and A. B. Gutman. 1959. Study of the paradoxical effects of salicylate in low, intermediate, and high dosage on the renal mechanisms for excretion of urate in man. J. Clin. Invest. 38: 1298.

10. Y ̈̈, T. F., L. Berger, and A. B. Gutman. 1961. Suppression of tubular secretion of urate by pyrazinamide in the dog. Proc. Soc. Exp. Biol. Med. 107: 905.

11. Rieselbach, R. E., L. B. Sorensen, W. D. Shelp, and T. H. Steele. 1970. Diminished renal urate secretion per nephron as a basis for primary gout. Ann. Intern. Med. $73: 359$.

12. Crowley, L. V., and F. T. Alton. 1968. Automated analysis of uric acid. Am. J. Clin. Pathol. 49: 285.

13. Keller, W. J. 1947. A rapid method for the determination of salicylates in serum or plasma. Am. J. Clin. Pathol. 17: 415.

14. Steele, T. H. 1969. Evidence for altered renal urate reabsorption during changes in volume of the extracellular fluid. J. Lab. Clin. Med. 74: 288.

15. Fanelli, G. M., Jr., D. Bohn, and S. Stafford. 1970. Functional characteristics of renal urate transport in the Cebus money. Am. J. Physiol. 218: 627.

16. Fanelli, G. M., Jr., D. Bohn, and S. Reilly. 1971. Renal urate transport in the chimpanzee. Am. J. Physiol. 220: 613.

17. Bennett, J. S., J. Bond, and I. Singer. 1972. Hyperuricemia in Hodgkin's disease. Ann. Intern. Med. 76: 751.

18. Yu, T. F., P. G. Dayton, and A. B. Gutman. 1963. Mutual suppression of the uricosuric effects of sulfinpyrazone and salicylate: a study in interactions between drugs. J. Clin. Invest. $42: 1330$.

19. Sirota, J. H., T. F. Yu, and A. B. Gutman. 1952. Effect of benemid ( $p$-[di- $n$-propylsulfamyl]-benzoic acid) on urate clearance and other discrete renal functions in gouty subjects. J. Clin. Invest. 31: 642.
20. Gutman, A. B., T. F. Yü, and L. Berger. 1959. Tubular secretion of urate in man. J. Clin. Invest. 38: 1778.

21. Bluestone, R., I. Kippen, J. R. Klinenberg, and M. W. Whitehouse. 1970. Effect of some uricosuric and antiinflammatory drugs on the binding of uric acid to human serum albumin in vitro. J. Lab. Clin. Med. 76: 85.

22. Sheikh, M. I., and J. V. Moller. 1968. Binding of urate to proteins of human and rabbit plasma. Biochim. Biophys. Acta. 158: 456.

23. Klinenberg, J. R., and I. Kippen. 1969. The binding of urate to plasma proteins determined by means of equilibrium dialysis. J. Lab. Clin. Med. 75: 503.

24. Simkin, P. A. 1972. Uric acid binding to serum proteins: differences among species. Proc. Soc. Exp. Biol. Med. $139: 604$.

25. Yu, T. F., J. H. Sirota, C. Berger, M. Halpern, and A. B. Gutman. 1957. Effect of sodium lactate infusion on urate clearance in man. Proc. Soc. Exp. Biol. Med. $96: 809$.

26. Diamond, H., E. Sharon, D. Holden, and A. Cacation. 1973. Renal handling of uric acid in sickle cell anemia. Clin. Res. In press. (Abstr.)

27. Weiner, I. M., J. A. Washington, II, and G. H. Mudge. 1960. On the mechanism of action of probenecid on renal tubular secretion. Bull. Johns Hopkins Hosp. 106: 333.

28. Gutman, A. B., P. G. Dayton, T. F. Yü, L. Berger, W. Chen, L. E. Sicam, and J. J. Burns 1960. A study of the inverse relationship between $\mathrm{pKa}$ and rate of renal excretion of phenylbutazone analogs in man and dog. $\mathrm{Am}$. J. Med. 29: 1017.

29. Postlewaite, A. E., and W. N. Kelley. 1971. Uricosuric effect of radiocontrast agents. Ann. Intern. Med. $74: 845$.

30. Mudge, G. H. 1971. Uricosuric action of cholecystographic agents. N. Engl. J. Med. $284: 929$. 